\title{
Simulation of Voltage/Current Waveforms and Contact Area of Pulsed Surface Discharge on Water
}

\author{
Tomohiro Furusato, Yota Yamamoto, Takuya Sakamoto, Kazushi Oura, \\ Yoshinobu Matsuda and Takahiko Yamashita \\ Nagasaki University \\ Graduate School of Engineering \\ Nagasaki, 852-8521, Japan
}

\begin{abstract}
Propagation of a surface discharge on water is evaluated using simulation results of the voltage/current waveforms and discharge contact area. Voltage/current waveforms are calculated using an exponential function which assumes the resistance of water decreases with increasing discharge contact area. A conductive disk having a given potential is used to model the discharge contact area and the temporal variation of the radius of the disk is discussed as the discharge propagation. The calculation in the current field is replaced by a calculation of the electrostatic field using the similarity between current and electrostatic fields. The calculation of the electrostatic field is conducted by a charge simulation method. The discharge phenomena are classified into two stages which are the breakdown of needle-to-water gap and the surface discharge propagation on water. The electrostatic field calculation at needle-to-water gap is performed to determine the initial discharge contact area. Expansion of the contact area in the creepage direction, is evaluated by the electric field calculation at the edge of the conductive disk. The expansion terminates when the field becomes lower than $26 \mathrm{kV} / \mathrm{cm}$. The velocity of the expansion increases with the applied voltage. The maximum contact radius increases with decreasing conductivity of water under the same applied voltage. The tendency of simulation results of the expansion of the contact area are consistent with the previous observation results of the discharge propagation.
\end{abstract}

Index Terms - surface discharges, water, simulation, pulsed power

\section{INTRODUCTION}

SURFACE discharge on water generated by a pulsed power has drawn attention as one of the advanced oxidation processes aiming the wastewater treatment $[1,2]$. The pulsed surface discharge plasma produces hydroxyl $(\mathrm{OH})$ radical having a high oxidation power, which generates an immediate chemical reaction to the organic compounds in water. [3]. The $\mathrm{OH}$ radicals are generated at the interface between the discharge plasma and water. Therefore, an estimation of the discharge contact area is of importance in an efficient wastewater treatment. In general, several visualization methods exist for the estimation of the radius of discharge channels. One is a laser Schlieren method which visualizes low-density region caused by thermal diffusion of discharge plasma $[4,5]$. The other is an observation method of the light emission from the discharge plasma with a high-speed camera [6]. In addition, ground-state $\mathrm{OH}$ radicals can be observed by a laser-induced fluorescence technique [3]. The above observation methods give us useful information; however, advanced

Manuscript received on 6 August 2018, in final form 11 November 2018, accepted xx Month 20yy. Corresponding author: T. Furusato. measurement technique and expensive equipment are necessary to realize those methods. Therefore, a simple method is desired for on-site analysis of the status of the surface discharge and for the optimum control of the equipment.

We developed a novel method which estimates the discharge area in contact with water, by analysing voltage and current waveforms [7]. The resistance of water part on which surface discharge propagates was calculated on the basis of Ohm's law. On the other hand, the resistance was calculated by assuming the radius of the contact area. The calculation was conducted using the similarity between the current field and the electrostatic field. The contact area was modeled as a circular conductive disk and the resistance was calculated from the contact area. The contact area was estimated by comparing the resistance obtained from the voltage/current waveform to the calculated one.

In our previous study, we dealt only with a specific case of the contact area under the condition where the discharge propagated maximum [7]. However, this study simulates the whole steps of the discharge propagating along the water surface. The discharge contact area and time-varying characteristics of the discharge contact area can be obtained by this simulation without observing the surface discharge. 


\section{EXPERIMENTAL SETUP AND SIMULATION MODEL}

\subsection{EXPERIMENTAL SETUP}

Experimental values of pulsed voltage and current are necessary in order to simulate a resistance variation of water by a surface discharge propagation. Figure 1 shows an experimental circuit of pulsed power supply and voltage and current measurement system. A tungsten needle electrode was set above the water surface. The charge stored in the $2 \mathrm{nF}$ capacitor, $\mathrm{C}_{0}$, is discharged by the selfspark gap switch. The gap between the needle tip and water was set approximately $1 \mathrm{~mm}$. The voltage at the needle tip was measured by a high-voltage probe (Iwatsu Co. Ltd., HV-P30) and the current was measured by current monitor (Pearson electronics Co. Ltd., model 4100). The water conductivity was adjusted by varying the potassium chloride concentration.

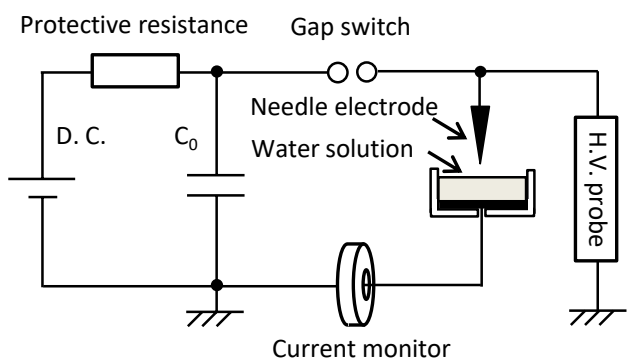

Figure 1. Experimental circuit and pulsed voltage and current measurement system.

\subsection{SIMULATION MODEL}

A discharge circuit model is introduced in order to discuss the voltage current characteristics. Figure 2 shows a circuit model after the air gap between the needle and water surface was shorted, which is following to the turn-on of the gap switch. The dominant voltage drops of the load are cathode fall voltage and the voltage drop across the water solution. This simulation does not take into account the inductance and resistance of the surface discharge, because they are negligibly small in comparison with the measured cathode fall voltage of around $1000 \mathrm{~V}$ [7]. We also neglected the effect of a thermalization of plasma on the cathode fall voltage because the discharge expands radially on the water cathode, which is significantly different from the case of metal cathode. However, the effect of the thermalization should be clarified in the future. The aqueous solution modeled by a parallel connection of the variable resistor and capacitor because the discharge contact area varies with the discharge propagation. The capacitance is negligible owing to the extremely small time constant of the water solution in this study (7.0 70 ns).

The discharge contact area can be estimated by comparing the calculated resistance with the measured one. Assuming the contact area, the resistance of water $R$ is calculated on the basis of a similarity between current field and electrostatic field, as shown in Figure 3. Our group previously applied the similarity to the surface discharge on wet polluted insulator under a lightning impulse voltage $[10,11]$. As stated above, the simplified equivalent circuit enables the treatment in the current field. Consequently, the calculation in the current field can be done in the electrostatic field taking into the similarity between the current and the electrostatic fields.

In general, the similarity leads the following equation:

$$
C R=\varepsilon \rho,
$$

where $\varepsilon$ is the permittivity, $\rho$ is the resistivity of water, and $C$ is the capacitance in the electrostatic field. The discharge contact area is simulated by a circular conductive disk having no thickness. Therefore, the $R$ in the current field is estimated by calculating $C$ in the electrostatic field. In the calculation of the electrostatic field, charged mirror disks are arranged so as to satisfy the boundary conditions in the current field.

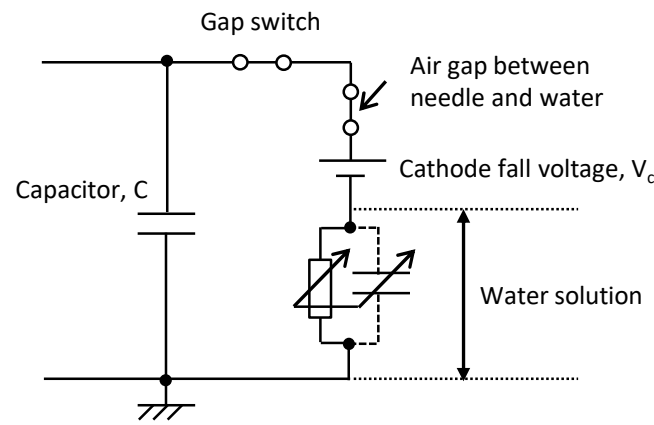

Figure 2. A simplified equivalent circuit of experimental system after turning on a gap switch.

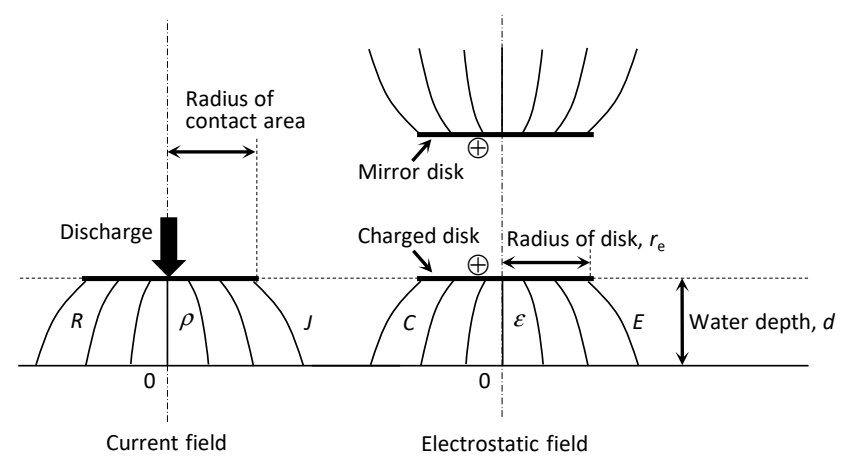

Figure 3. A model of equivalent discharge contact area using disk and mirror electrodes with charge simulation method.

\section{RESULTS AND DISCUSSION \\ 3.1 DISCHARGE CONTACT AREA AND RESISTANCE OF WATER}

Figure 4 shows the relation between the calculated $R$ and the contact radius. The value of $R$ decreases with increasing the conductivity of water. Consequently, the discharge contact area is estimated by comparing the calculated $R$ and the measured resistance. The detailed measurement method of the resistance of water using measured voltage and current waveforms will be explained in Section 3.2.

Figure 5 shows voltage and current waveforms at the same voltage peak of $15 \mathrm{kV}$ under various water conductivity. The width of voltage pulse decreases with the water conductivity, while the current pulse becomes higher. The rapid decrease of the voltage under the relatively high water conductivity is explained to be caused by a large number of the discharge branching and the rapid discharge propagation. The large 


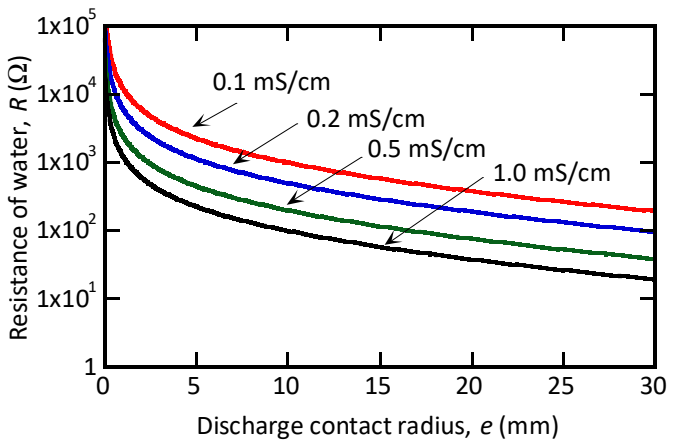

Figure 4. Calculated resistance versus contact radius under different conductivity of water (water depth: $10 \mathrm{~mm}$ ).

current pulse leads to the high electric field along the water surface and results in the rapid discharge propagation [12]. According to the equivalent circuit (Figure 2), the measured resistance $R(t)$ is obtained by Equation (1) based on Ohm's law:

$$
R(t)=\frac{v(t)-V_{c}}{i(t)}
$$

where $v(t)$ is the applied voltage, $i(t)$ is the current, and $V_{\mathrm{c}}$ is the cathode fall voltage. The detailed measurement method of $V_{\mathrm{c}}$ is described in the literature [7]. Figure 6 shows the $R(t)$ obtained by Equation (2). The $R(t)$ rapidly decreases with time immediately initiation of the discharge. Meanwhile, the $R(t)$ gradually increases after the value indicated minimum value. At the moment of the minimum value of $R(t)$, the discharge propagation takes the maximum which was validated by the previous study [7].

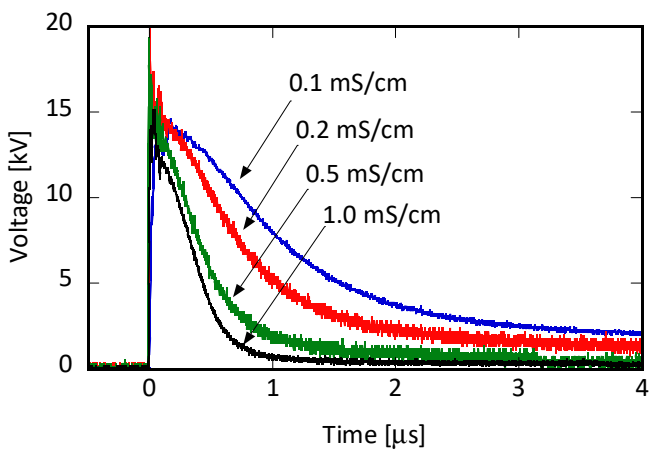

(a) Voltage waveforms

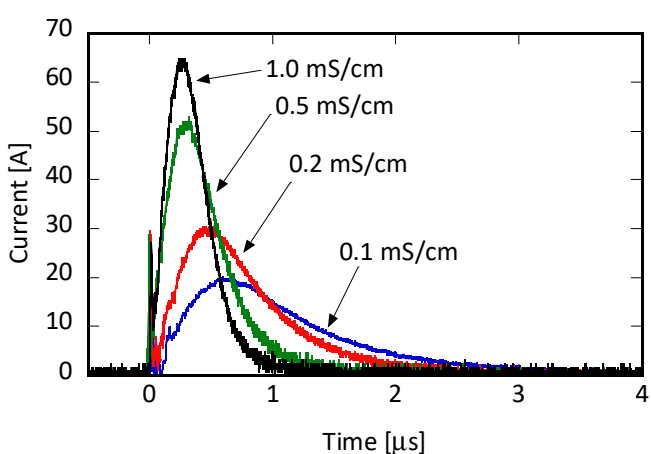

(b) Current waveforms.

Figure 5. Measured voltage and current waveforms under the same voltage peak.

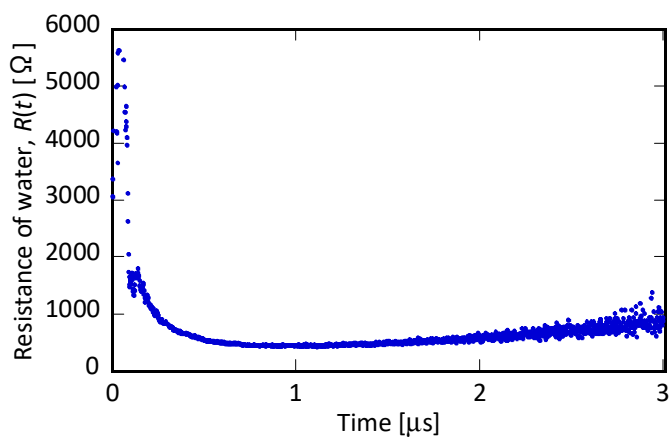

Figure 6. Variation of measured resistance of water with time for water conductivity of $0.1 \mathrm{mS} / \mathrm{cm}$ and applied voltage of $15 \mathrm{kV}$.

\subsection{REPRODUCTION OF VOLTAGE/CURRENT WAVEFORMS}

We assume the following equation with exponential function relevant to the resistance of water in order to simulate the discharge propagation:

$$
R_{S}(t)=\left(R_{0}-R_{\min }\right) e^{-B t}+R_{\min },
$$

where $R_{\mathrm{s}}(t)$ is the simulated resistance of water, $R_{0}$ is the value of resistance of water at the moment when the discharge is initially in contact with the water, $R_{\min }$ is minimum value of resistance, and $B$ is the fitting parameter. In Equation (3), we neglect the variation of the plasma resistance because the resistance is supposed much smaller than that of the water. The estimation method of the $R_{0}$ will be explained in sub-section 3.3. The $R_{\min }$ is given by the minimum value of the measured resistance of water that corresponds to the moment of the maximum discharge propagation (see Figure 6). The simulation of $R_{\mathrm{s}}(t)$ is fitted to measurements by a nonlinear least square analysis, as shown in Figure 7 . The simulation result of equation well fits to the measurement value.

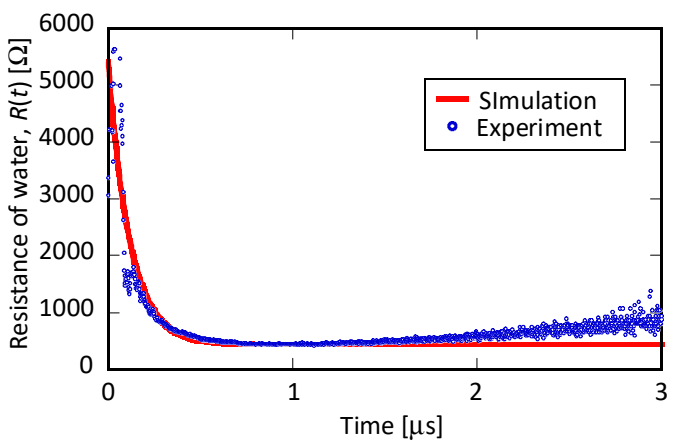

Figure 7. Measured resistance for water conductivity of $0.1 \mathrm{mS} / \mathrm{cm}$ and applied voltage of $15 \mathrm{kV}$ and simulated resistance variation.

The voltage was simulated by following equation based on the simplified experimental circuit (see Figure 2):

$$
V_{n}=\left(V_{n-1}-V_{c}\right) \exp \left(\frac{-\Delta t}{R_{S}\left(t_{n-1}\right) C}\right)+V_{c},
$$

where the $V_{\mathrm{n}}$ and $V_{\mathrm{n}-1}$ are discrete value of voltage with an interval of $\Delta t$ and the capacitance of $C$ which connected in 
parallel with the variable resistance of $R_{\mathrm{s}}(t)$ is $2 \mathrm{nF}$. A sketch of the discrete values of voltage is shown in Figure 8 . The $V_{\mathrm{n}}$ is calculated by Equation (4) corresponding the $R_{\mathrm{s}}\left(t_{\mathrm{n}-1}\right)$. A time constant of $R_{\mathrm{S}}(t) C$ varies whenever the voltage is calculated at each discrete point. Provided that, the initial value of this voltage simulation is used the measured applied voltage. The simulation of the current was performed by assigning the discrete values of the voltage to Equation (3). The simulation result of voltage and current with $\Delta t$ equal to $2 \mathrm{~ns}$ is shown in Figure 9, which are well fit to the measurements. The simulated waveforms will be used the simulation of the discharge propagation in Section 3.5.

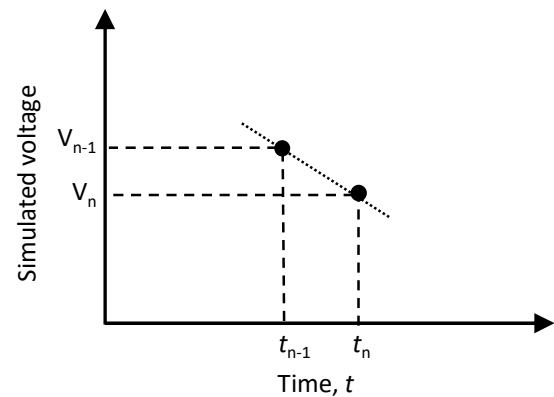

Figure 8. Sketch of the discrete value of the simulated voltage. $V_{\mathrm{n}-1}$ and $V_{\mathrm{n}}$ corresponding to Equation (4).

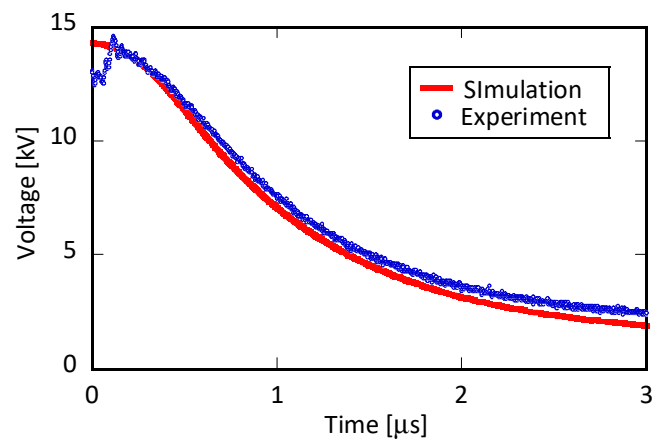

(a) Voltage waveform

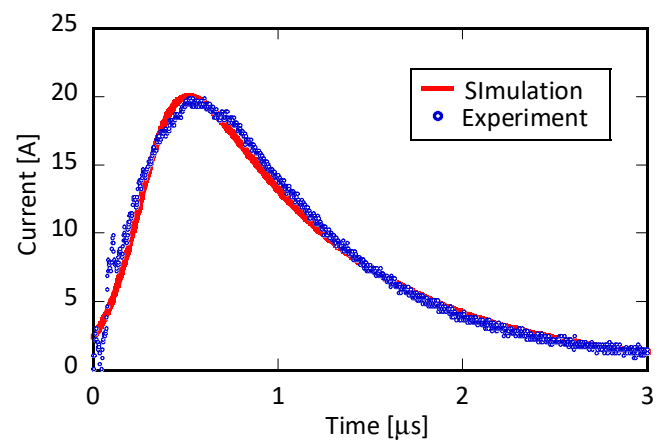

(b) Current waveform

Figure 9. Measured and simulated voltage and current waveforms for water conductivity of $0.1 \mathrm{mS} / \mathrm{cm}$ and applied voltage of $15 \mathrm{kV}$.

Figure 10 shows the $R_{\min }$ which corresponds to the maximum discharge propagation as a function of the applied voltage. The
$R_{\min }$ exponentially decreases with the applied voltage and depends on the water conductivity, whereas the $B$ linearly increases with the applied voltage independent of the water conductivity, as shown in Figure 11. The $B$ is associated with the propagation characteristics of the surface discharge on water; however, detailed relation is not well understood at this time. The detailed discussion will be reported in the next paper.

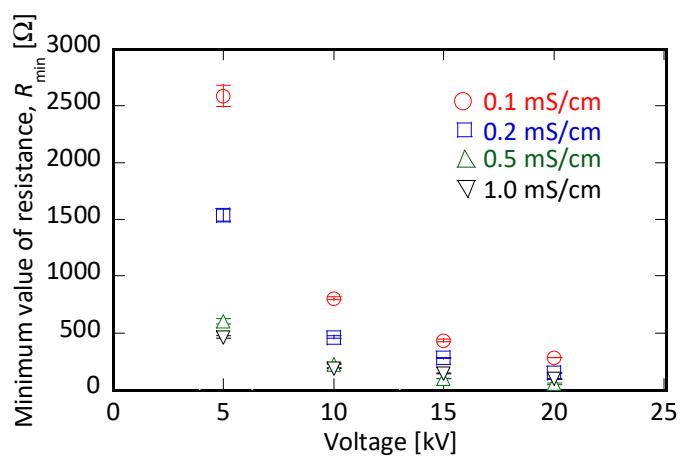

Figure 10. Measured minimum value of resistance versus applied voltage under different conductivity of water.

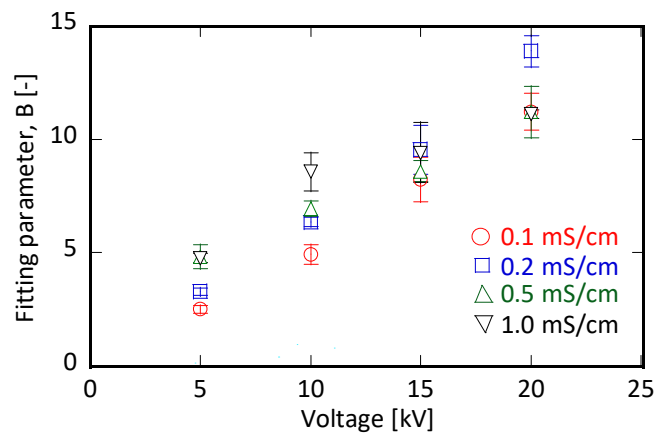

Figure 11. Fitting parameter versus applied voltage under different conductivity of water.

\subsection{DISCHARGE INITIATION BETWEEN NEEDLE ELECTRODE AND WATER}

An initial condition of resistance of water $R_{0}$ is necessary to simulate the propagation of discharge. Figure 12 shows an illustration of the start of the diffusive glow discharge in contact with water. As found in $[8,13]$ for the case of needle-to-water electrode, the pulsed discharge plasma ignites in the form of diffusive glow under the initial state. The sketched area between needle and water in Figure 12 means an active region. The threshold value of the active region in atmospheric air is 26 $\mathrm{kV} / \mathrm{cm}$ according to [14]. The electric field just above the water under the various applied voltage ranging from 5 to $20 \mathrm{kV}$ were calculated by a software, Ansoft Maxwell (Ansoft Japan Co., Ltd.). The water is assumed conductive material such as metal in the electric field calculation. Figure 13 shows calculation results of the electric field separated $0.1 \mu \mathrm{m}$ from the metal plane as a function of the creepage distance from just below the needle tip. The value of field drastically decreases with the distance. The diameter of the rod electrode and gap distance were set at $1 \mathrm{~mm}$. The maximum value of the radius where the electric field strength is higher than $26 \mathrm{kV} / \mathrm{cm}$ is determined as 
a radius of initial discharge contact area. Consequently, the value of resistance of water at the moment when the discharge is initially in contact with the water, $R_{0}$, is determined uniquely from the relation between the calculated resistance and discharge contact area (see Figure 4).

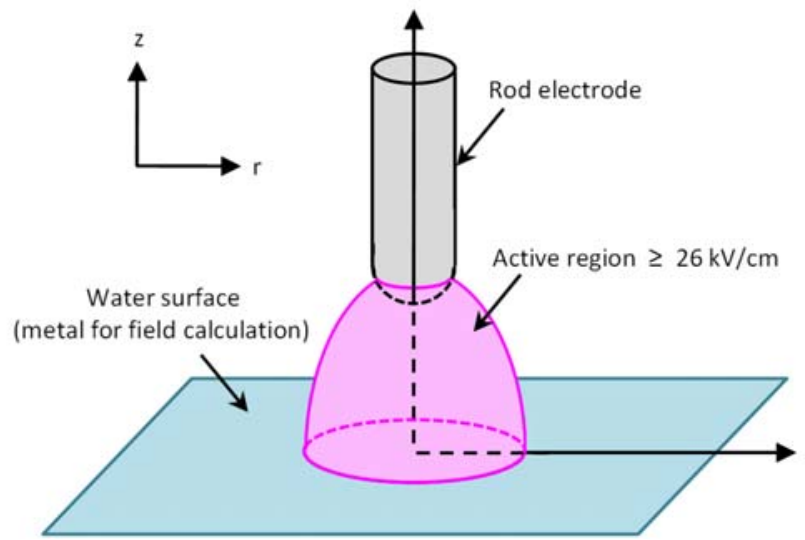

Figure 12. Illustration of the active region between rod electrode and water under the discharge initiation (pre-breakdown) phase.

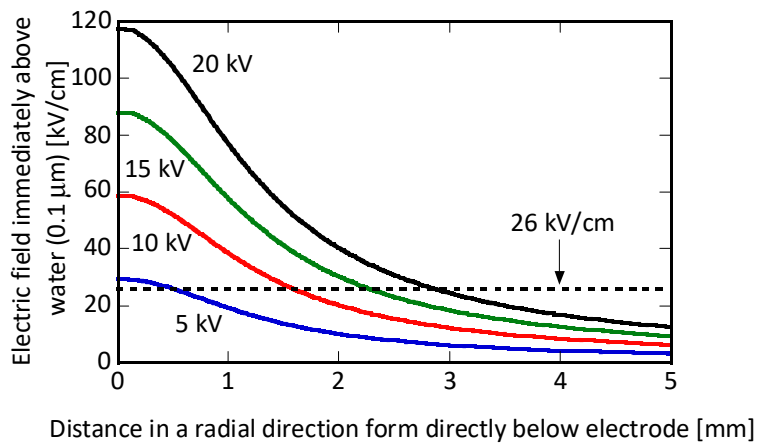

Figure 13. Field calculation just above water $(0.1 \mu \mathrm{m})$ as a function of distance in a radial direction under the various applied voltage.

\subsection{SIMULATION OF DISCHARGE PROPAGATION}

The surface discharge propagation was evaluated by the field calculations at the edge of the charged disk. Figure 14 shows illustration of the propagation processes of the charged disk. The radius including the electric field in the creepage direction $E_{\mathrm{r}}$ greater equal to $26 \mathrm{kV} / \mathrm{cm}$ is determined as newly propagated contact radius. The above propagation process is repeated until the field at the edge of contact radius is less than $26 \mathrm{kV} / \mathrm{cm}$.

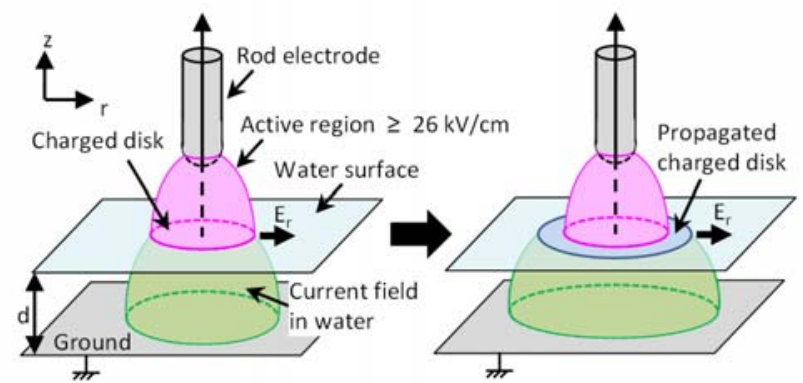

Figure 14. Illustration of the expansion process of charged disk by field calculation in the creepage direction.
The calculation method is as follows. The initial discharge contact radius $e_{0}$ is determined by an active region as stated in sub-section 3.3. The value subtracted the cathode fall voltage from the initial value of simulated potential (see Figure 9 (a)) is used as the potential $V_{0}$ on the disk of $e_{0}$ in radius. The calculation of charge $Q_{0}$ on the disk of $e_{0}$ in radius is necessary to calculate the electric field in the creepage direction. In general, the potential at $\mathrm{P}(r, z)$ by charged and mirror disks is expressed as follows [15]:

$$
\begin{gathered}
V=\sum_{i=0}^{n} V_{i}=\sum_{i=0}^{n} \frac{Q_{i}}{4 \pi \varepsilon_{0} e} \arctan \frac{\sqrt{2} e}{\sqrt{D_{i}+\sqrt{D_{i}^{2}+4 e^{2}\left(z-d_{i}\right)^{2}}}}, \\
D_{i}=r^{2}+\left(z-d_{i}\right)^{2}-e^{2},
\end{gathered}
$$

where $i$ is index at each charged and mirror disks, $n$ is number of the charged and mirror disks, $Q_{\mathrm{i}}$ is the charge, $e$ is the radius of the charged disk, $d_{\mathrm{i}}$ is the distance from vertical axis to the charged disk, and $V$ is the potential at $\mathrm{P}(r, z)$. The positional relation of charged and mirror disks is shown in Figure 15. The electric field $E_{\mathrm{r}}$ can be calculated by Equation (6) [15]:

$$
\begin{aligned}
& E_{r}=\sum_{i=0}^{n} E_{i} \\
& =\sum_{i=0}^{n} \frac{k Q_{i}}{4 \pi \varepsilon_{0} e} \frac{Y}{1+Y^{2}} \frac{r}{\sqrt{\left(r_{i}^{2}+\left(z-d_{i}\right)^{2}-e^{2}\right)+4 e^{2}\left(z-d_{i}\right)^{2}}} \\
& Y=\frac{1}{\sqrt{2} e} \times \\
& \sqrt{r^{2}+\left(z-d_{i}\right)^{2}-e^{2}+\sqrt{r^{2}+\left(z-d_{i}\right)^{2}-e^{2}+4 e^{2}\left(z-d_{i}\right)^{2}}}
\end{aligned}
$$

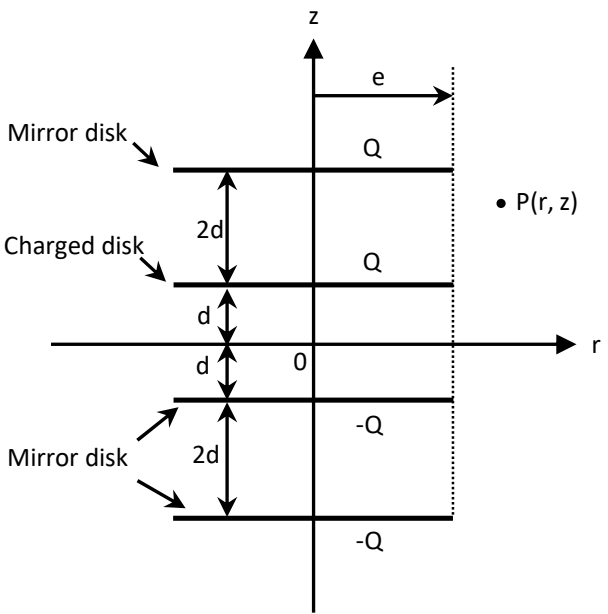

Figure 15. Positional relation between charged disk and mirror disk on z-r coordinate.

Figure 16 shows detailed algorism of the calculation process. The field calculation is performed on the inspection points that are arranged at intervals of $10 \mu \mathrm{m}$ in the creepage direction, as shown in Figure 17. The charge $Q_{0}$ at the initial contact of the discharge is determined by assuming $V_{0}$ for $V$ at $e_{0}$. Provided that, the electric field at the inspection point $10 \mu \mathrm{m}$ apart form charged disk is determined as the electric field at the edge of the charged disk. After getting a new contact area, the resistance is calculated in order to determine the potential at the expanded charged disk from the variations of the resistance and the voltage. 


\subsection{SIMULATION RESULTS OF DISCHARGE PROPAGATION}

Figure 18 shows the time-varying characteristic of the simulated contact radius under the same conductivity of water of $0.1 \mathrm{mS} / \mathrm{cm}$ by the proposed method. The slopes at each applied voltage on Figure 18 mean velocity of the surface discharge. The slope increases with the increase of the applied voltage because the field $E_{\mathrm{r}}$ at the edge of the initial contact area also increases with the voltage. The saturated value of the timevarying characteristic is determined as a maximum contact radius that corresponds to the stop of the discharge. Figure 19

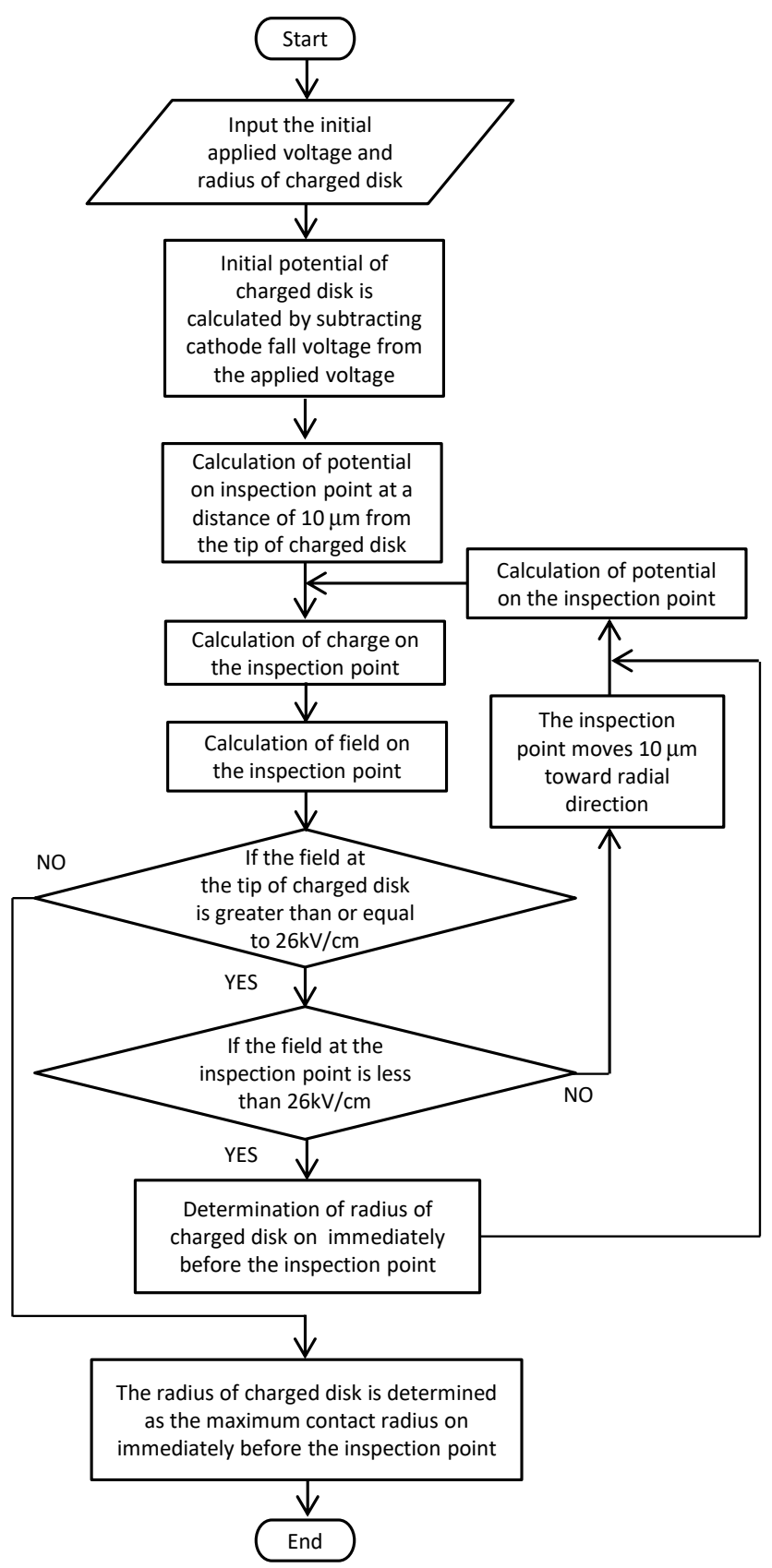

Figure 16. Proposed algorithm for the calculation of the propagating charged disk. shows the maximum contact radius as a function of the applied voltage under different conductivity of water. The trend of increase of contact radius with applied voltage is the same at any conductivity; however, the value of radius decreases with the increase of the conductivity under the same applied voltage. The dependence of maximum discharge length on the water conductivity is consistent with the discharge observation results in the literature [16].

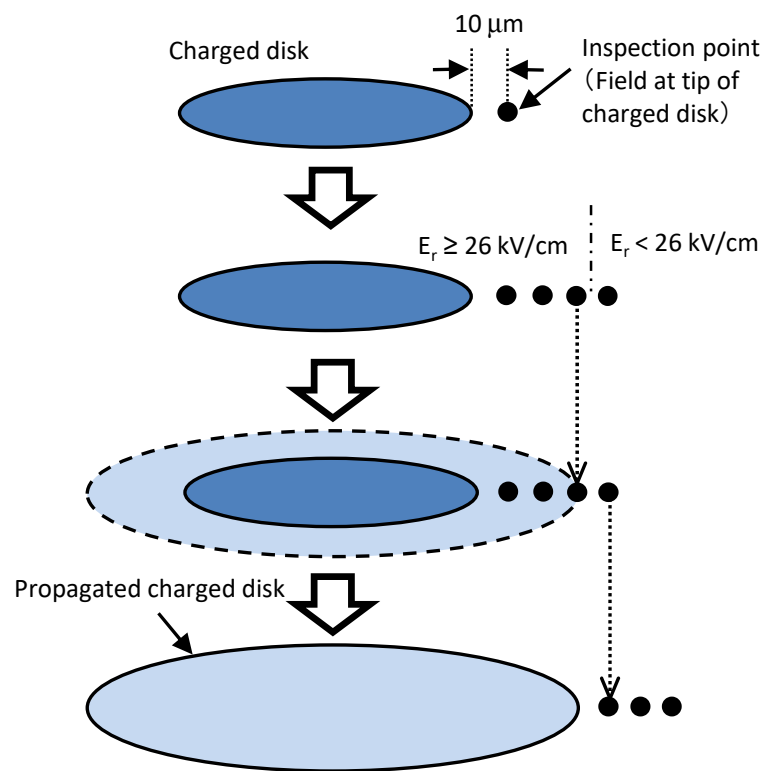

Figure 17. Calculation process of field on inspection point and determination of propagated charged disk.

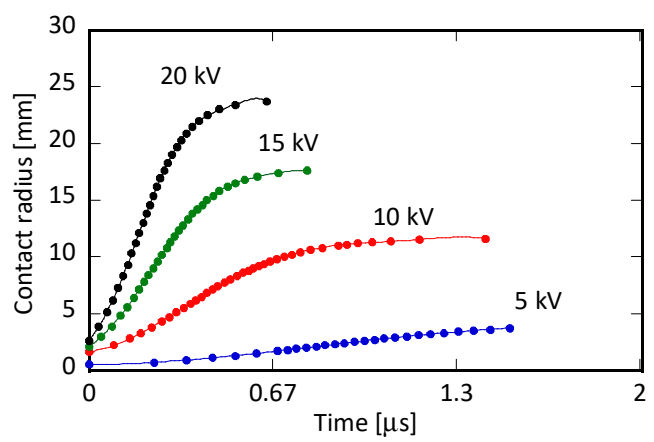

Figure 18. Calculated contact radius as a function of applied voltage of 5 , 10,15 , and $20 \mathrm{kV}$ under the same conductivity of water of $0.1 \mathrm{mS} / \mathrm{cm}$.

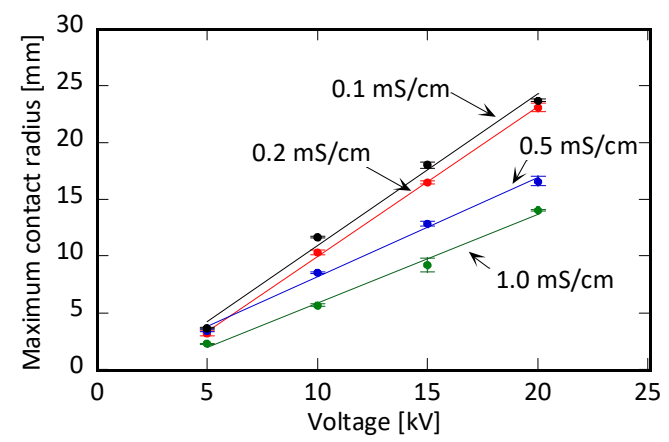

Figure 19. Maximum contact radius as a function of applied voltage under different conductivity of water. 


\section{CONCLUSIONS}

This paper simulates voltage/current waveforms using proposed discharge contact area and evaluates the propagation of a pulsed surface discharge on water. The simulation is based on the similarity between current and electrostatic fields and conducted using the charge simulation method. The electric field at the edge of the contact area is also calculated by charge simulation method. Whether the electric field at the end of the contact area is higher or lower compared with the ionization field of the air decides termination of discharge. The results are summarized as follows.

1) Voltage/current waveforms were reproduced using an approximation of resistance of water and an analysis of transient phenomena, where cathode fall voltage is taken into account. The variation of resistance was approximated by an exponential function. As a result, the voltage/current waveforms were well reproduced.

2) The discharge phenomena are classified into two phase which are the breakdown of needle-to-water gap and the surface discharge propagation on water. The electrostatic field calculation of rod-plane electrode configuration was performed to determine an initial contact area. The expansion of the contact area was also evaluated by the electric field at the end of the contact area.

3) The time-varying characteristic of the discharge contact radius was simulated by our proposed method. The characteristic indicates the velocity of surface discharge increases with the applied voltage peak.

\section{ACKNOWLEDGMENT}

This work was partially supported by JSPS KAKENHI Grant-in-Aid for Scientific Research (C) Grant Number $16 \mathrm{~K} 06231$.

\section{REFERENCES}

[1] P. Lukes and B. R. Locke, "Plasmachemical oxidation processes in a hybrid gas-liquid electrical discharge reactor,” J. Phys. D. Appl. Phys., vol. 38, no. 22, pp. 4074-4081, 2005.

[2] M. Sato, T. Tokutake, T. Ohshima, and A. T. Sugiarto, "Aqueous phenol decomposition by pulsed discharges on the water surface," IEEE Trans. Ind. Appl., vol. 44, no. 5, pp. 1397-1402, 2008.

[3] S. Kanazawa, H. Kawano, S. Watanabe, T. Furuki, S. Akamine, R. Ichiki, T. Ohkubo, M. Kocik, and J. Mizeraczyk, "Observation of $\mathrm{OH}$ radicals produced by pulsed discharges on the surface of a liquid," Plasma Sources Sci. Technol., vol. 20, no. 3, pp. 1-8, 2011.

[4] T. Furusato, H. Tanoue, S. Katsuki, M. Hara, and H. Akiyama, "Prebreakdown process of a negative nanosecond pulsed discharge in supercritical carbon dioxide," Appl. Phys. Express, vol. 7, no. 2, pp. 1-3, 2014.

[5] T. Furusato, T. Ihara, S. Kameda, T. Kiyan, S. Katsuki, M. Hara, and H. Akiyama, "Fractal analysis of positive pulsed streamer patterns in supercritical carbon dioxide," IEEE Trans. Plasma Sci., vol. 40, no. 10 PART 1, pp. 2425-2430, 2012.

[6] E. M. Van Veldhuizen and W. R. Rutgers, "Pulsed positive corona streamer propagation and branching," J. Phys. D. Appl. Phys., vol. 35, no. 17, pp. 2169-2179, 2002.

[7] T. Furusato, D. Obata, Y. Yota, and Y. Takahiko, “A New Evaluation Method of Contact Area at Interface between Pulsed Surface Discharge and Water," IEEE Trans. Plasma Sci., vol. 46, no. 6, pp. 2079-2084, 2018.
[8] T. Yamashita, M. Hisao, O. Yoshihiro, and O. Tsuyoshi, "Current density at the local discharge tip on an electrolytic surface," IEEE Trans. Electr. Insul., vol. 25, no. 6, pp. 1104-1110, 1990.

[9] P. Bruggeman, E. Ribel, A. Maslani, J. Degroote, A. Malesevic, R. Rego, J. Vierendeels, and C. Leys, "Characteristics of atmospheric pressure air discharges with a liquid cathode and a metal anode," Plasma Sources Sci. Technol., vol. 17, pp. 1-11, 2008.

[10] H. Matsuo, T. Yamashita, and W. D. Shi, "Electrical contact between a local discharge on an electrolytic solution and the solution surface," IEEE Trans. Dielectr. Electr. Insul., vol. 7, no. 3, pp. 360-365, 2000.

[11] H. Matsuo, T. Yamashita, and T. Fujishima, "Shape of contacting surface between an electrolytic solution and local discharge on it," IEEE Trans. Dielectr. Electr. Insul., vol. 10, no. 4, pp. 634-640, 2003.

[12] T. Furusato, T. Sadamatsu, Y. Matsuda, and T. Yamashita, "Streamer Branching and Spectroscopic Characteristics of Surface Discharge on Water Under Different Pulsed Voltages," IEEE Trans. Plasma Sci., vol. 45, no. 4, pp. 711-717, 2017.

[13] P. Bruggeman, J. L. Walsh, D. C. Schram, C. Leys, and M. G. Kong, "Time dependent optical emission spectroscopy of sub-microsecond pulsed plasmas in air with water cathode," Plasma Sources Sci. Technol., vol. 18, no. 4, pp. 1-5, 2009.

[14] I. Gallimberti, “The mechanism of the long spark formation," Le J. Phys. Colloq., vol. 40, no. C7, pp. C7-193-C7-250, 1979.

[15] M. Akazaki, K. Nishijima, and S. Saito, "Calculation of three dimensional axisymmetric fields by charge simulation method," Electr. Eng. Japan, vol. 98, no. 4, pp. 351-358, 1978.

[16] N. S. Midi, R. Ohyama, and S. Yamaguchi, "Underwater current distribution induced by spark discharge on a water surface," J. Electrostat., vol. 71, no. 4, pp. 823-828, 2013.

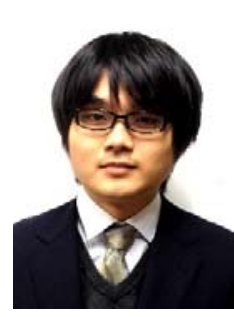

Tomohiro Furusato (S'13-M'14) was born in Kagoshima, Japan, in 1988. He received the B. E., M. E., and $\mathrm{Ph}$. D. degrees from Kumamoto University, Kumamoto Japan, in 2011, 2012, and 2014, respectively. He was with the Japan Society for the Promotion of Science, Kumamoto University, from 2013 to 2014, as a Research Fellow. Since 2014, he has been an Assistant Professor with the Graduate School of Engineering, Nagasaki University, Nagasaki, Japan. His research interests are pulsedpower, surface discharge, and discharge phenomena in supercritical fluids.

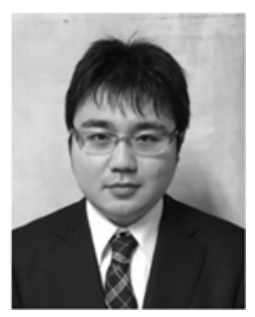

Yota Yamamoto was born in Aichi, Japan, in 1995. $\mathrm{He}$ received the B.E. degree from Nagasaki University, Nagasaki, in 2016, where he is currently pursuing the M.E. degree. His current research interests include surface discharge phenomena on water.

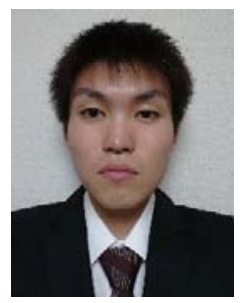

Takuya Sakamoto was born in Oita, Japan, in 1995. $\mathrm{He}$ received the B.E. degree from Nagasaki University, Nagasaki, in 2017, where he is currently pursuing the M.E. degree. His current research interests include simulation of a surface discharge on water.

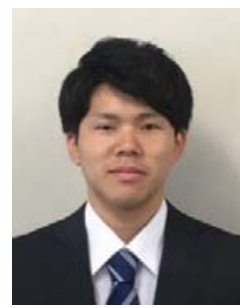

Kazushi Oura was born in Nagasaki, Japan, in 1995. $\mathrm{He}$ received the B.E. degree from Nagasaki University, Nagasaki, in 2017, where he is currently pursuing the M.E. degree. His current research interests include spectroscopic measurements of surface discharge phenomena on water. 


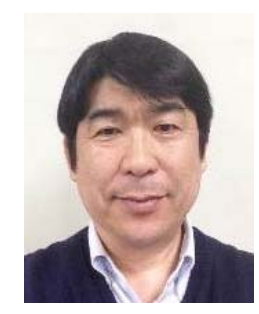

Yoshinobu Matsuda was born in Miyazaki, Japan, in 1960. He received B. S. degree from Kyoto

University, Kyoto Japan in 1982, and M. E. and Ph.

D. degrees from Kyushu University, Fukuoka

Japan in 1984 and 1987, respectively. After

working as an Assistant Researcher with the

Graduate School of Science and Engineering,

Kyushu University from 1987 to 1988 , he joined

the Department of Electrical and Electronic

engineering, Nagasaki University as a Lecturer.

Since 1990, he has been an Assistant Professor with the Graduate School of Engineering, Nagasaki University, Nagasaki, Japan. His research interests are plasma diagnostics, plasma surface interaction, and engineering application of plasmas, plasma processing of thin films in particular.

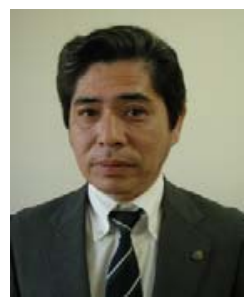

Takahiko Yamashita (M'00) was born in Fukuoka, Japan, in 1957. He received the B.E., M.E. and D.E. degrees from Kyushu University in 1980, 1982 and 1985, respectively. He has been working in Nagasaki University, Nagasaki, Japan since 1985. He is a Professor of Graduate School of Engineering and a Vice President of Nagasaki University. He is a senior member of Institute of Electrical Engineers Japan. His research interests discharge phenomena. 